\title{
Outcomes of Endovascular Thrombectomy for Basilar Artery Occlusion
}

\author{
Christopher R. Pasarikovski (D), Houman Khosravani, Leodante da Costa, \\ Chinthaka Heyn, Stefano M. Priola, Jerry C. Ku, Sandra E. Black, \\ David J. Gladstone, Victor X. D. Yang
}

\begin{abstract}
Background and Purpose: Large prospective observational studies have cast doubt on the common assumption that endovascular thrombectomy (EVT) is superior to intravenous thrombolysis for patients with acute basilar artery occlusion (BAO). The purpose of this study was to retrospectively review our experience for patients with BAO undergoing EVT with modern endovascular devices. Methods: All consecutive patients undergoing EVT with either a second-generation stent retriever or direct aspiration thrombectomy for BAO at our regional stroke center from January 1, 2013 to March 1, 2019 were included. The primary outcome measure was functional outcome at 1 month using the modified Rankin Scale (mRS) score. Multivariable logistic regression was used to assess the association between patient characteristics and dichotomized mRS. Results: A total of 43 consecutive patients underwent EVT for BAO. The average age was 67 years with $61 \%$ male patients. Overall, 37\% (16/43) of patients achieved good functional outcome. Successful reperfusion was achieved in $72 \%$ (31/43) of cases. The median (interquartile range) stroke onset to treatment time was 420 (270-639) minutes (7 hours) for all patients. The procedure-related complication rate was 9\% (4/43). On multivariate analysis, posterior circulation Alberta stroke program early computed tomography score and Basilar Artery on Computed Tomography Angiography score were associated with improved functional outcome. Conclusion: EVT appears to be safe and feasible in patients with BAO. Our finding that time to treatment and successful reperfusion were not associated with improved outcome is likely due to including patients with established infarcts. Given the variability of collaterals in the posterior circulation, the paradigm of utilizing a tissue window may assist in patient selection for EVT. Magnetic resonance imaging may be a reasonable option to determine the extent of ischemia in certain situations.
\end{abstract}

RÉSUMÉ : Résultats associés à la thrombectomie endovasculaire dans le cas de patients victimes d'une occlusion de l'artère basilaire. Contexte et objectif : D'importantes études observationnelles prospectives ont remis en question la supposition commune suivant laquelle la thrombectomie endovasculaire (TEV) est supérieure à la thrombolyse intraveineuse dans le cas de patients victimes d'une occlusion de l'artère basilaire $(\mathrm{OAB})$ de nature aigüe. L'objectif de cette étude est donc de passer en revue de façon rétrospective notre expérience avec des patients victimes d'une $\mathrm{OAB}$ qui ont été soumis à une TEV au moyen de dispositifs endovasculaires récents. Méthodes : Nous avons inclus dans cette étude tous les patients vus consécutivement qui ont subi, à la suite d'une OAB, une TEV, que ce soit avec une endoprothèse retriever de seconde génération (second-generation stent retriever) ou par aspiration directe. À noter que ces patients choisis ont été admis dans notre centre régional de soins de l'AVC du $1^{\mathrm{er}}$ janvier 2013 au $1^{\mathrm{er}}$ mars 2019 . Le principal résultat mesuré a été le niveau d'autonomie fonctionnelle au bout d'un mois. Pour ce faire, nous avons fait appel à l'échelle modifiée de Rankin (EMR). Nous avons en outre effectué une analyse de régression logistique multivariée afin d'évaluer l'association entre les caractéristiques des patients et des résultats d'analyse dichotomique au moyen de l'EMR. Résultats : Au total, 43 patients ont été soumis à une TVE en raison d'une OAB. Leur âge moyen était de 67 ans ; de plus, $61 \%$ étaient de sexe masculin. De façon générale, $37 \%$ des patients (16/43) sont parvenus à des résultats satisfaisants en matière d'autonomie fonctionnelle. Une reperfusion réussie a été accomplie dans $72 \%$ des cas (31/43). Le délai médian entre le début des AVC et le moment où un traitement a été prodigué a été de 420 minutes (7h) (écart interquartile : 270-639) pour tous les patients. Le taux de complication en lien avec cette intervention a été quant à lui de $9 \%$ (4/43). Enfin, en fonction d'une analyse multivariée, les résultats au Alberta Stroke Program Early Computed Tomography Score (ASPECTS) pour la circulation postérieure et au Basilar Artery on Computed Tomography Angiography (BATMAN) ont été associés à des niveaux d'autonomie fonctionnelle améliorés. Conclusion : Il semble donc que la TEV est à la fois sécuritaire et réalisable chez des patients victimes d'OAB. Notre constat suivant lequel les délais d'obtention d'un traitement et une reperfusion réussie ne sont pas

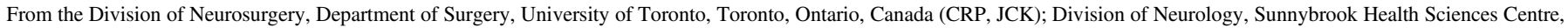

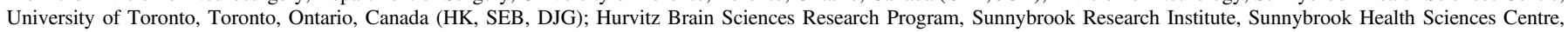

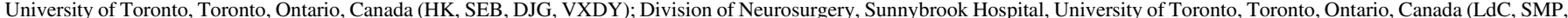

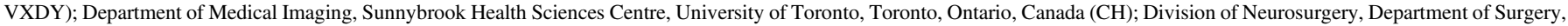
Health Sciences North, Sudbury, Ontario, Canada (SMP); Norther Ontario School of Medicine, Sudbury, Ontario, Canada (SMP)

Received December 7, 2019. Final Revisions Submitted January 14, 2020. Date of Acceptance February 20, 2020.

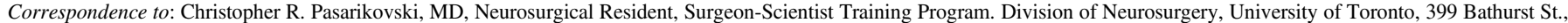
WW 4-427 Toronto, ON M5T 2S8, Canada. Email: chris.pasarikovski@mail.utoronto.ca 
associés à une amélioration de l'autonomie fonctionnelle des patients peut probablement être attribué au fait d'avoir inclus des patients avec des infarctus dits «complétés » (established infarcts). Étant donné la variabilité des vaisseaux collatéraux dans la circulation postérieure, il se peut que le modèle d'intervention reposant sur les dommages encourus au tissu cérébral (paradigm of utilizing a tissuewindow) soit utile dans la sélection des patients en vue d'une TEV. Qui plus est, des examens d'IRM pourraient constituer une option raisonnable pour déterminer dans certaines situations l'étendue d'un AVC.

Keywords: Stroke, Basilar, Endovascular thrombectomy, Posterior circulation

doi:10.1017/cjn.2020.51

Can J Neurol Sci. 2020; 47: 479-485

\section{INTRODUCTION}

Acute basilar artery occlusion (BAO) is associated with a significant rate of death and disability. ${ }^{1}$ Large prospective observational studies did not support the superiority of endovascular thrombectomy (EVT) over intravenous thrombolysis (IVT) for patients with acute BAO and others demonstrated poor clinical outcome despite successful recanalization. ${ }^{2,3}$ The Acute Basilar Artery Occlusion: Endovascular Interventions versus Standard Medical Treatment (BEST) trial was the first randomized controlled trial designed to evaluate the efficacy of EVT for patients with BAO. ${ }^{4}$ The trial was terminated early due to progressive loss of equipoise among recruiting physicians resulting in high crossover rates. The only other randomized trial for acute basilar occlusion assessed the safety and efficacy of intraarterial urokinase in 16 patients and was halted early due to poor recruitment. ${ }^{5}$ Collectively, these studies highlight the clinical challenges pertaining to the management of BAO. ${ }^{6}$

Given the heterogeneous group of patients undergoing EVT for BAO, stroke societies currently describe the benefits of EVT in BAO as uncertain. ${ }^{7,8}$ The aim of this study is to audit our local experience for patients with acute BAO undergoing EVT with modern endovascular devices, as part of a quality improvement initiative for our regional stroke center. The descriptive analysis explored predictors of good functional outcome.

\section{Methods}

\section{Patient Characteristics}

Research ethics board approval was obtained for this singlecenter retrospective observational study. All consecutive patients undergoing EVT for BAO at our center, a high EVT volume ( $>125$ cases/year) regional stroke center serving the north and east Greater Toronto Area, from January 1, 2013 to March 1, 2019 were included. There were no exclusion criteria. During this period, as anterior circulation large vessel occlusion EVT went from clinical trials' phase to standard of care, our institution did not have specific EVT inclusion/exclusion criteria for patients with BAO, which was not an uncommon scenario in many centers. Patient selection for EVT was made on an individual basis by the consulting stroke neurologist and neurointerventionalist. Generally, EVT was considered in patients $>18$ years, prestroke modified Rankin Scale (mRS) score 0-2, with no intracranial hemorrhage (ICH) or evidence of extensive bilateral brainstem ischemia. The authors do not use a time window cutoff and considered patients $>24$ hours from ictus in certain situations.

Patient baseline characteristics retrieved included age, sex, stroke risk factors (hypertension, smoking, dyslipidemia, coronary heart disease, diabetes, and atrial fibrillation), and antithrombotic therapy. Administration of intravenous tissue plasminogen activator (tPA), symptom onset to puncture time, and stroke etiology according to the trial of ORG 10172 in acute stroke treatment classification were recorded. ${ }^{9}$ Baseline National Institutes of Health Stroke Scale (NIHSS) score was calculated on arrival by the stroke team.

All patients underwent computed tomography (CT) angiography (CTA) before and after EVT (within 24 hours). Imaging features retrospectively obtained include any evidence of ICH after EVT, posterior circulation Alberta Stroke Program Early CT Score (pc-ASPECTS), length of thrombus, Basilar Artery on Computed Tomography Angiography (BATMAN) score, and early (<24 hour) reocclusion. ${ }^{10,11}$ pc-ASPECTS is a 10-point scale where points are lost for infarction of the thalami, occipital lobes, cerebellar hemispheres, midbrain, and pons as observed on noncontrast CT. The BATMAN score is a 10-point CTA-based grading system utilized to assess the extent of basilar occlusion and the presence of posterior communicating artery collateralization. Points are distributed as: one point if either vertebral artery is patent, one point for each proximal posterior cerebral artery, one point for each patent segment of the basilar artery (proximal, middle, and distal), and two points for each of the posterior communicating arteries.

\section{Endovascular Thrombectomy}

Procedures were performed at our regional stroke center with anesthesia coverage. The primary technique used was determined by the treating neurointerventionalist and included either a second-generation stent retriever or direct contact aspiration. Intra-arterial thrombolysis was utilized in certain instances if the clot could not be traversed or significant residual stenosis/thrombus remained. We recorded the primary device used as the treatment technique, along with the number of attempts before recanalization.

\section{Outcome Measures}

The primary outcome measure was functional outcome at 1 month. The mRS score was used to define dichotomized functional outcome, as measured by stroke physicians. A mRS of 0-3 was considered a good outcome, and mRS 4-6 was considered a poor outcome as defined in the BASICS registry. ${ }^{2}$ Secondary outcome measures included reperfusion status as determined on the final angiographic run during EVT and the incidence of procedure-related complications. The modified treatment in cerebral infarction (mTICI) score was used, with $2 \mathrm{~B}-3$ defined as successful reperfusion and 0-2A defined as unsuccessful. Stroke-onset time was defined as the onset of symptoms as 
per the patient or witnesses and consistent with the clinical diagnosis of BAO by the treating stroke physician. Prodromal symptoms such as transient ischemic attack or minor stroke in the hours/days leading to the current event were not considered as the stroke-onset time. For example, if patients experienced episodes of dizziness/vertigo and diplopia one day prior and now presented with severe neurological deficit, the time of onset would be the severe deficit and subsequent imaging confirming BAO.

\section{Statistical Analysis}

All variables (patient baseline, clinical, imaging, and treatment) were compared between patients with good (mRS 0-3) and poor (mRS 4-6) functional outcomes. All continuous variables were recorded as means with interquartile range, and categorical variables were recorded as counts with percentages. For continuous variables, an independent sample $t$-test or Mann-Whitney $\mathrm{U}$ test was used to compare the two groups. The Chi-squared or Fishers' exact tests were used to compare categorical variables depending on number of samples in each quadrant. The threshold for significance was defined as $\mathrm{p}<0.05$. For those values found to be significant, logistic regression analysis was performed with odds ratios (OR) and 95\% confidence intervals (95\% CI). Statistical analysis was done using SPSS version 25.

\section{ReSUltS}

A total of 43 consecutive patients underwent EVT for BAO with no patients excluded from the analysis. The average age (standard deviation) was 67 years (16) with 61\% (26/43) male patients. Overall, 37\% (16/43) of patients achieved good functional outcome (mRS $0-3)$ at 1 month (Table 1). The 1-month mortality rate was $33 \%(16 / 43)$. Successful reperfusion (mTICI 2B-3) was achieved in $72 \%$ (31/43) of cases. In patients with successful reperfusion, 39\% (12/31) achieved good function outcome and in patients with unsuccessful reperfusion, $33 \%$ (4/12) achieved good functional outcome.

The procedure-related complication rate was $9 \%$ (4/43). In three patients, intraoperative ICH occurred, as observed with active contrast extravasation (two subarachnoid hemorrhages with intraventricular extension and one intraparenchymal hemorrhage). All three patients had extensive CT evidence of established infarcts (pc-ASPECTS $\leq 6$ ) before EVT and none received intravenous or intra-arterial thrombolysis. The fourth patient had a large midbrain infarct prior to EVT and developed hemorrhagic transformation on postoperative (24 hours) imaging. He received intravenous tPA with time to EVT puncture of 255 minutes.

The median (interquartile range) stroke onset to treatment time was 420 (270-639) minutes ( $7 \mathrm{~h}$ ) for all patients. Good functional outcome was achieved in $33 \%$ (7/21) of those patients treated $<6$ hours of stroke onset and in $40 \%(9 / 22)$ of patients treated $>6$ hours of stroke onset. The mean onset to treatment time for all patients with good and poor functional outcomes was 674 and 624 minutes, respectively. The longest onset to treatment time was 48 hours in a patient with postural neurological changes that required ongoing blood pressure augmentation, who also achieved good functional outcome. The most common BAO etiology was basilar atherosclerotic disease $(60 \%)$, followed by cardioembolism (26\%) and undetermined (12\%).
On univariate analysis, baseline NIHSS, pc-ASPECTS, and BATMAN scores were all associated with improved functional outcome $(p<0.05)$. Length of occlusion and stroke etiology were not found to be significantly associated with improved functional outcome. Stroke onset to puncture time, successful reperfusion, and the administration of tPA were also not found to be significantly associated with outcome $(\mathrm{p}=0.68)$ (Figure 1). There was also no difference between patients treated with stent retrievers and contact aspiration. On multivariate analysis, only pc-ASPECTS and BATMAN scores were found to be significant $(\mathrm{p}<0.05)$.

\section{Discussion}

Our single-center retrospective study of 43 patients observed good functional outcome in $37 \%$ of patients, successful recanalization achieved in $72 \%$ of patients, with a periprocedural complication rate of $9 \%$. Despite functional improvement in a subset of patients, a majority of patients with successful recanalization did not achieve good outcomes, which is consistent with previously described series. Intriguingly, a portion of patients without recanalization also achieved good outcome in short-term follow-up highlighting challenges in predicting outcome based on measurable parameters in this fulminant type of stroke.

Our study has several limitations. First, this was a retrospective observational study, and patient selection criteria for EVT could be subject to selection biased. There was no standardization for patient and treatment selection.

The BASICS registry is the largest prospective observational study done to assess outcomes and treatment responses for 619 patients with BAO. ${ }^{2}$ They observed that overall $32 \%$ of patients had favorable outcome (mRS 0-3) at 1 month, and that there was no difference between those patients receiving IVT and EVT. This study cast doubt on the assumption that IAT is superior to IVT in patients with BAO. More recently, ENDOSTROKE subsequently examined 148 patients with BAO undergoing EVT (84\% second-generation stent retrievers) and observed $42 \%$ of patients had good outcome (mRS $0-3$ ), with $35 \%$ mortality rate. ${ }^{3}$ They found however that the presence of successful reperfusion was not correlated to outcome, again underscoring the need to search for reasons of futility of recanalization in patients with BAO. ${ }^{12}$ Our findings are consistent with the notion that vessel recanalization alone is insufficient and that some patients will even have adequate recovery without vessel recanalization.

Two multicenter randomized controlled trials were undertaken comparing the safety and efficacy of EVT plus best medical management compared to best medical management alone for patients with acute BAO. ${ }^{4,13}$ The BEST trial was terminated early due to progressive loss of equipoise resulting in high crossover rates. This is not an uncommon scenario in EVT centers. Despite the early termination of the trial, those patients who were treated with EVT had significantly better outcomes. ${ }^{6}$ In the as-treated analysis, $47 \%$ of patients who underwent EVT had a good outcome (mRS 0-3) at 90 days compared to $24 \%$ in the standard medical therapy group $(\mathrm{p}=0.008)$. Symptomatic ICH was reported in $8 \%$ of the intervention group. ${ }^{14}$ The BASICS trial recruitment continues. ${ }^{6}$

Our findings of good function outcome in $37 \%$ of patients and successful reperfusion in $72 \%$ are in agreement with more recent 
Table 1: Patient baseline characteristics, imaging findings, and treatment techniques dichotomized with respect to good (mRS 0-3) and poor ( $m R S ~ 4-6)$ functional outcome. Univariate analysis was used to examine for differences between the groups

\begin{tabular}{|c|c|c|c|c|}
\hline Characteristic & All patients $(\mathrm{N}=43)$ & Good outcome $(N=16)$ & Poor outcome $(\mathrm{N}=27)$ & p-Value \\
\hline Age (years) & $67(57-97)$ & $65(56-76)$ & $68(58-79)$ & 0.6 \\
\hline Sex (male) & $26(61 \%)$ & $9(21 \%)$ & $17(40 \%)$ & 0.7 \\
\hline Baseline NIHSS score (mean, interquartile range) & $18(6-28)$ & $10(3.75-13)$ & $22(16-28)$ & $<0.001 *$ \\
\hline Intravenous thrombolysis (tPA) & $15(35 \%)$ & $7(16 \%)$ & $8(19 \%)$ & 0.4 \\
\hline $\begin{array}{l}\text { Onset to treatment time (mean, interquartile } \\
\text { range) minutes }\end{array}$ & $643(270-643)$ & $674(198-762)$ & $624(300-639)$ & 0.8 \\
\hline \multicolumn{5}{|l|}{ Risk factors } \\
\hline Hypertension & $27(63 \%)$ & $10(23 \%)$ & $17(40 \%)$ & 0.9 \\
\hline Dyslipidemia & $17(40 \%)$ & $6(14 \%)$ & $11(26 \%)$ & 0.8 \\
\hline $\mathrm{T} 2 \mathrm{DM}$ & $12(28 \%)$ & $3(7 \%)$ & $9(21 \%)$ & 0.3 \\
\hline AFIB & $8(19 \%)$ & $5(12 \%)$ & $3(7 \%)$ & 0.1 \\
\hline $\mathrm{CAD}$ & $14(33 \%)$ & $5(12 \%)$ & $9(21 \%)$ & 0.9 \\
\hline Smoker & $5(12 \%)$ & $1(2 \%)$ & $4(9 \%)$ & 0.4 \\
\hline \multicolumn{5}{|l|}{ Stroke classification } \\
\hline Atherosclerotic & $26(60 \%)$ & $8(19 \%)$ & $18(42 \%)$ & \multirow[t]{4}{*}{0.2} \\
\hline Cardioembolism & $10(23 \%)$ & $3(7 \%)$ & $7(16 \%)$ & \\
\hline Dissection & $5(12 \%)$ & $4(9 \%)$ & $1(2 \%)$ & \\
\hline Undetermined & $2(5 \%)$ & $1(2 \%)$ & $1(2 \%)$ & \\
\hline \multicolumn{5}{|l|}{ Imaging } \\
\hline pc-ASPECTS (mean, interquartile range) & $7.44(6-10)$ & $9.25(8.75-10)$ & $6.37(5-8)$ & $<0.001 *$ \\
\hline BATMAN score (mean, interquartile range) & $7(6-8)$ & $8.19(8-9)$ & $6.30(1-7.75)$ & $<0.001 *$ \\
\hline Clot length $(\mathrm{cm})$ & $1.3(0.8-1.9)$ & $1.1(0.7-1)$ & $1.4(0.8-2)$ & 0.3 \\
\hline Intracranial hemorrhage & $4(9 \%)$ & 0 & $4(9 \%)$ & 0.1 \\
\hline \multicolumn{5}{|l|}{ Reperfusion } \\
\hline 2B-3 (successful) & $32(75 \%)$ & $9(21 \%)$ & $23(53 \%)$ & \multirow[t]{2}{*}{0.7} \\
\hline $0-2 \mathrm{~A}$ (unsuccessful) & $11(25 \%)$ & $7(16 \%)$ & $4(9 \%)$ & \\
\hline \multicolumn{5}{|l|}{ Treatment technique } \\
\hline Stent retriever & $21(49 \%)$ & $8(19 \%)$ & $13(30 \%)$ & \multirow[t]{3}{*}{0.1} \\
\hline Aspiration & $13(30 \%)$ & $2(5 \%)$ & $11(26 \%)$ & \\
\hline IAT & $4(9 \%)$ & $3(7 \%)$ & $1(2 \%)$ & \\
\hline Number of attempts & $1.4(1-2)$ & $1.1(0.8-1)$ & $1.6(1-2)$ & 0.2 \\
\hline
\end{tabular}

Abbreviations: pc-ASPECTS $=$ Posterior circulation Alberta Stroke Program Early CT Score; BATMAN = Basilar Artery on Computed Tomography Angiography; NIHSS = National Institutes of Health Stroke Scale; T2DM = type 2 diabetes mellitus; AFIB = atrial fibrillation; CAD = coronary artery disease; IAT $=$ intra-arterial thrombolysis.

EVT series for patients with BAO. ${ }^{15-19}$ The authors report a complication rate of $9 \%$, which is in line with that of the BEST trial which reported symptomatic ICH rate of $8 \%$ in the intervention arm. ${ }^{14}$ We observed that only baseline NIHSS score, pcASPECTS, and BATMAN score were significantly associated with good outcome on univariate analysis, and only the baseline pc-ASPECTS and BATMAN score were significant on multivariate logistic regression analysis. As observed in the ENDSTROKE study, we did not find that successful reperfusion or decreased symptom onset to treatment time was associated with improved functional outcome. We hypothesize several reasons for these findings.
With respect to stroke onset to treatment time, defining accurate symptom onset in BAO is challenging, as some patients experience a prodromal phase of symptoms before the basilar occludes. $^{20}$ Thus, the standard anterior circulation definition of "last seen normal" time does not always convey the same implications for BAO patients. This may lead to prolonged recorded symptom-onset times. Furthermore, the posterior circulation's unique collateralization may allow for longer time interval for intervention. Lindsberg et al. hypothesized that a thrombus will cause a drop in pressure in the basilar artery, leading to backflow into the basilar via the posterior communicating arteries, maintaining vital brainstem perforators if the clot 


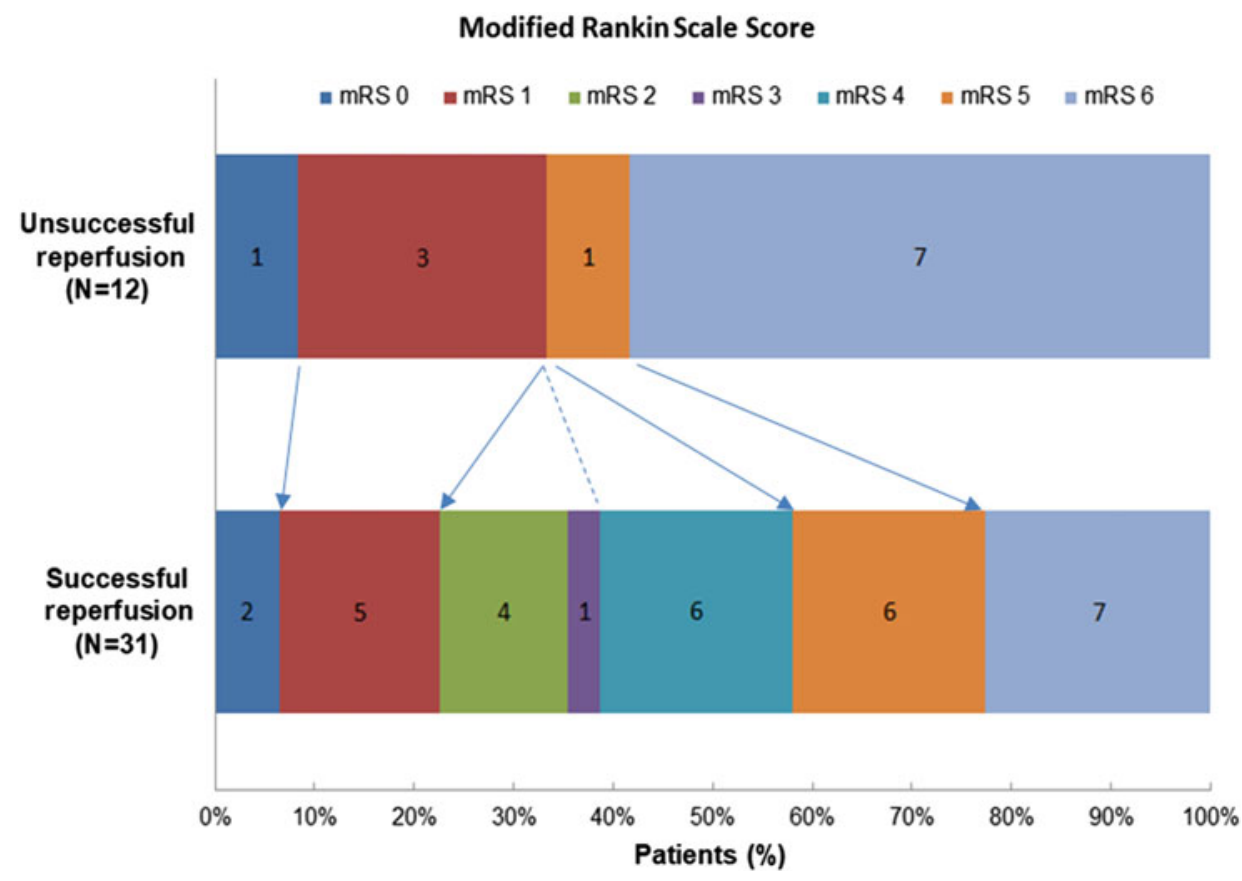

Figure 1: Scores on the modified Rankin Scale as a function of EVT reperfusion status. Successful reperfusion (mTICI 2B-3) was achieved in $72 \%$ patients (31/43). The presence of successful reperfusion was not found to be associated with dichotomized good functional outcome (dash line).

does not enlarge (Figure 2). ${ }^{21}$ The extent of brainstem ischemia and recovery after BAO depends on the integrity of vascular collateralization to vital brainstem structures not yet infarcted. However, any stepwise growth of thrombus will put these structures at risk. Intervention in these patients could be beneficial, within an extended time window after the onset of symptoms.

Second, the finding that successful reperfusion was not associated with improved functional outcome could be due to treating a significant number of patients with already established disabling brainstem infarcts, in which revascularization is futile (Figure 3). We found that $40 \%$ of patients treated $>6$ hours of stroke onset had good functional outcome, compared with just $33 \%$ in those treated $<6$ hours of onset, which is contrary to established paradigms of early reperfusion improving outcome. Our finding that only pc-ASPECTS score on multivariable analysis was associated with outcome supports the hypothesis that patients with evidence of significant brainstem ischemic before EVT have poor outcome. Puetz et al. showed that pc-ASPECTS score independently predicted death and functional outcome from patients in the BASICS registry. ${ }^{22}$ It is notable that pc-ASPECTS based on CTA source images may have further sensitivity in detecting already infarcted tissue. ${ }^{23}$ Furthermore, hyperacute rapid magnetic resonance imaging (MRI) protocols may also be informative in-patient selection and/or hemorrhagic risk stratification in patients who are being revascularized. In the ENDOSTROKE study, they found that use of magnetic resonance before EVT also predicted clinical outcome. We speculate that it is unlikely there is a large ischemic penumbra in the brainstem, particularly to midbrain/pontine structures supplied by single perforators/circumflex arteries, and hence diffusion weighted imaging (DWI) changes observed will not be reversed. The basilar artery vascular territory has been largely excluded
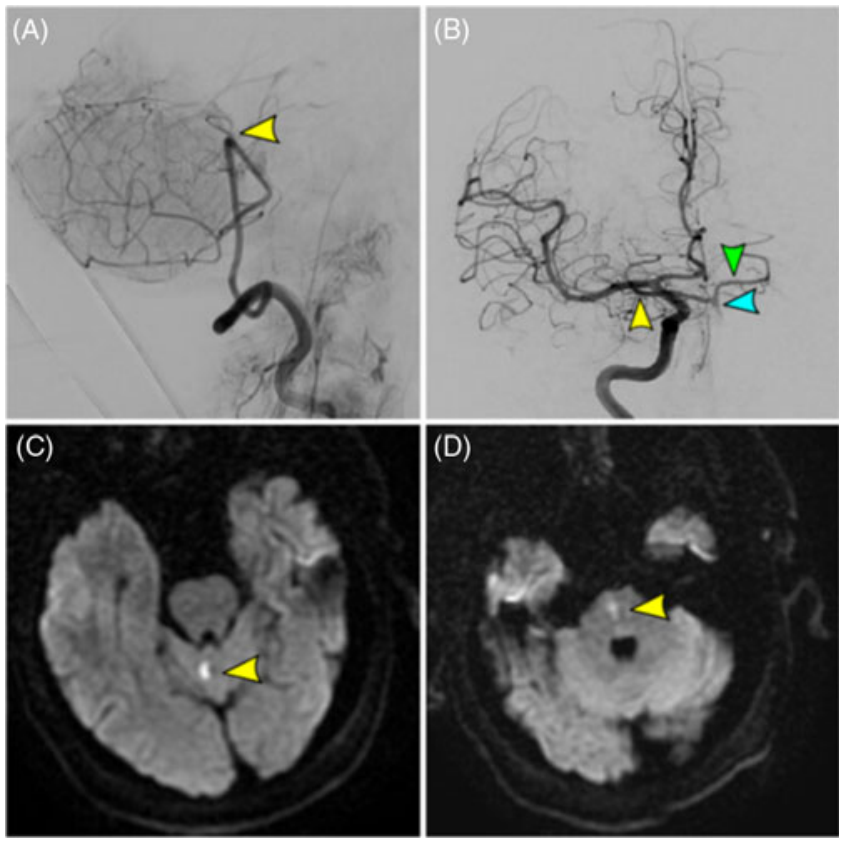

Figure 2: Acute basilar occlusion in a 67-year-old male with failed recanalization (TICI 0 ) and $m R S O$ at 1 month. (A) Vertebral artery injection (final angiographic run) showing proximal basilar occlusion (yellow arrow) with failed recanalization. (B) Right internal carotid artery injection showing retrograde flow through the right posterior communicating artery (yellow arrow) into the basilar artery (blue arrow) and contralateral posterior cerebral artery (green arrow). (C/D) Small cerebellar and medulla infarcts as observed on DWI MRI.

Abbreviations: $T I C I=$ thrombolysis in cerebral infarction, $m R S=$ modified Rankin Scale, DWI = diffusion-weighted imaging, MRI = magnetic resonance imaging. 

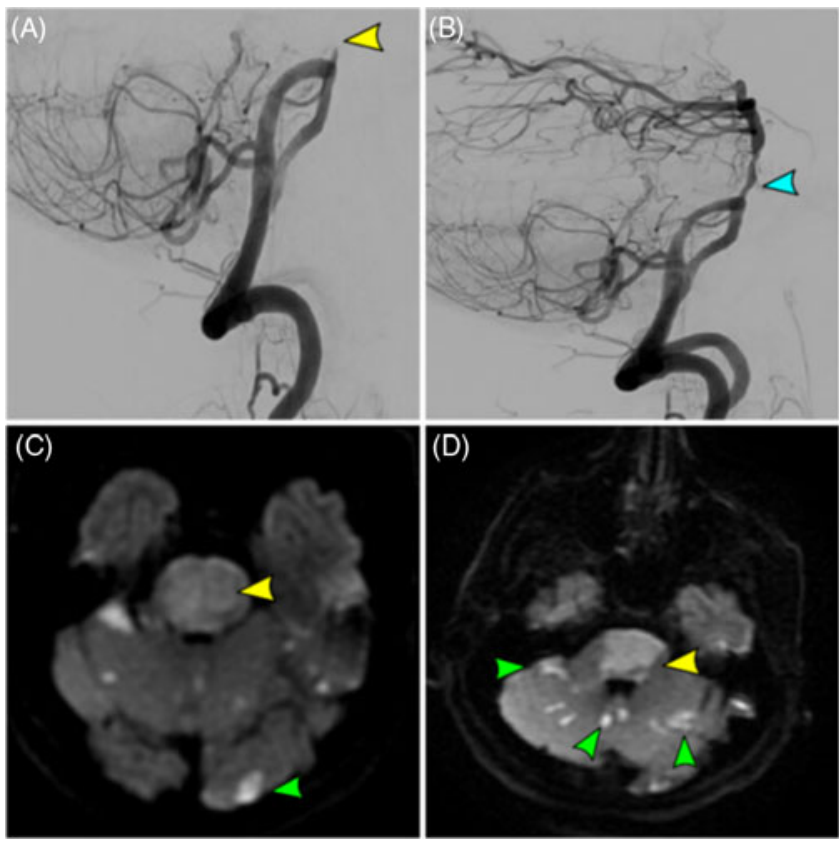

Figure 3: Acute basilar occlusion with successful recanalization (TICI $2 B$ ) in a 79-year-old female with time to puncture of 4.5 hours and discharge mRS 5. (A) Vertebral artery injection showing proximal basilar occlusion (yellow arrow). (B) Final angiographic run demonstrating TICI $2 B$ reperfusion with significant ongoing mid-basilar stenosis (blue arrow). (C/D) Large diffuse cerebellar (green arrows) infarcts and extensive medullary infarcts (yellow arrows) as observed on DWI MRI.

Abbreviations: $T I C I=$ thrombolysis in cerebral infarction, $m R S=$ modified Rankin Scale, DWI = diffusion-weighted imaging, MRI = magnetic resonance imaging.

from the ischemic penumbra literature, and the limited data suggest that no penumbra can exist in the pons after occlusion of internal arteries. ${ }^{21}$ The anatomical location of posterior circulation infarcts also plays a role in outcome. Lee $e t a l$. showed that diffusion restriction in the pons was independently associated with early neurological deterioration, while other locations (midbrain, thalamus, cerebellum, and medulla) were not independent predictors. ${ }^{24}$

Collectively, our finding and those from the literature demonstrate that patient selection for EVT for BAO remains an important challenge. We hypothesize that strict time of symptom-onset thresholds for EVT inclusion may exclude patients that would benefit from EVT. Sterbian et al. showed that in the absence of extensive baseline ischemia, EVT up to 48 hours was effective and pc-ASPECTS was the single most important prognostic factor and independent of time to treatment. ${ }^{25}$ It is likely that thrombus within the basilar artery will progress over time, and EVT could prevent clot propagation and eventual infarction of vital structures in patients with salvageable brainstem. Furthermore, mechanism of thrombus (cardioembolic vs. atherosclerotic) impacts on clinical progression and recognition, rate of tissue infarction, and EVT-related technical challenges. The paradigm of utilizing a tissue window as oppose to a time window for EVT patient selection was on display in the Thrombectomy 6 to 24 Hours after Stroke with a Mismatch between Deficit and Infarct and Thrombectomy for Stroke at 6 to 16 Hours with Selection by Perfusion Imaging trials, and this concept could also be expanded to the posterior circulation, especially given the posterior circulation collateralization. ${ }^{26}$

The findings of the present study have solidified the authors opinion that hard time window cutoffs for EVT consideration in BAO may not be advantageous. Furthermore, patients with poor neurological examination and non-contrast CT evidence of extensive brainstem infarcts are typically not considered for EVT. At that point, discussions are held with the family regarding the observed irreversible brainstem injury and futility of revascularization. In cases where the clinical examination is poor and noncontrast CT imaging does not clearly show brainstem ischemia, the authors have started utilizing rapid MRI DWI imaging typically within 30 minutes to determine the extent of brainstem injury.

\section{Conclusion}

EVT is safe and feasible for patients with acute BAO. Patient selection for thrombectomy for basilar occlusion remains a challenge. Given the unique and variable collateral anatomy, the paradigm of utilizing a tissue window as oppose to a time window for patient selection could be advantageous. The authors finding (along with findings in the literature) that time to treatment and successful reperfusion were not associated with improved outcome is likely due to including patients with established infarcts, and MRI imaging may be a reasonable option to determine the extent of ischemia in certain situations.

\section{CONFLiCT OF INTEREST}

All authors declare no conflicts of interest.

\section{AuthorshiP}

Study concept and design: CRP, HK, LdC, CH, SMP, JCK, SEB, DG, VXDY; Acquisition, analysis, or interpretation of data: CRP, HK, LdC, CH, SMP, JCK, SEB, DG, VXDY; Drafting the article: CRP, HK, LdC, DG, VXDY; Administrative, technical, or material support: CRP, HK, LdC, CH, VXDY; Statistical analysis: CRP, HK, DG, VXDY; Study supervision: VXDY.

\section{REFERENCES}

1. Lindsberg PJ, Soinne L, Tatlisumak T, et al. Long-term outcome after intravenous thrombolysis of basilar artery occlusion. JAMA 2004;292(15):1862-6.

2. Schonewille WJ, Wijman CA, Michel P, et al. Treatment and outcomes of acute basilar artery occlusion in the Basilar Artery International Cooperation Study (BASICS): a prospective registry study. Lancet Neurol. 2009;8(8):724-30.

3. Singer OC, Berkefeld J, Nolte $\mathrm{CH}$, et al. Mechanical recanalization in basilar artery occlusion: the ENDOSTROKE study. Ann Neurol. 2015;77(3):415-24

4. Liu X, Xu G, Liu Y, et al. Acute basilar artery occlusion: endovascular Interventions versus Standard Medical Treatment (BEST) Trial-Design and protocol for a randomized, controlled, multicenter study. Int J Stroke 2017;12(7):779-85.

5. Macleod MR, Davis SM, Mitchell PJ, et al. Results of a multicentre, randomised controlled trial of intra-arterial urokinase in the treatment of acute posterior circulation ischaemic stroke. Cerebrovasc Dis. 2005;20(1):12-7.

6. World Stroke Congress Abstracts, 2018 - Late breaking submissions. Int J Stroke 2018;13(2_suppl):225-44.

7. Powers WJ, Rabinstein AA, Ackerson T, et al. 2018 Guidelines for the Early Management of Patients With Acute Ischemic Stroke: a Guideline for Healthcare Professionals From the American Heart 
Association/American Stroke Association. Stroke 2018;49(3): e46-e110.

8. Boulanger JM, Lindsay MP, Gubitz G, et al. Canadian Stroke Best Practice Recommendations for Acute Stroke Management: Prehospital, Emergency Department, and Acute Inpatient Stroke Care, 6th Edition, Update 2018. Int J Stroke 2018;13(9):949-84.

9. Adams HP, Jr., Bendixen BH, Kappelle LJ, et al. Classification of subtype of acute ischemic stroke. Definitions for use in a multicenter clinical trial. TOAST. Trial of Org 10172 in Acute Stroke Treatment. Stroke 1993;24(1):35-41.

10. Puetz V, Sylaja PN, Coutts SB, et al. Extent of hypoattenuation on CT angiography source images predicts functional outcome in patients with basilar artery occlusion. Stroke 2008;39(9):2485-90.

11. Alemseged F, Shah DG, Diomedi M, et al. The Basilar Artery on Computed Tomography Angiography Prognostic Score for Basilar Artery Occlusion. Stroke 2017;48(3):631-7.

12. Lindsberg PJ, Strbian D. Recanalization of basilar artery occlusion. Ann Neurol. 2015;78(5):832-3.

13. van der Hoeven EJ, Schonewille WJ, Vos JA, et al. The Basilar Artery International Cooperation Study (BASICS): study protocol for a randomised controlled trial. Trials 2013;14:200.

14. Liu X, Dai Q, Ye R, et al. Endovascular treatment versus standard medical treatment for vertebrobasilar artery occlusion (BEST): an open-label, randomised controlled trial. Lancet Neurol. 2019.

15. Verstraete M, Bounameaux H, de Cock F, Van de Werf F, Collen D. Pharmacokinetics and systemic fibrinogenolytic effects of recombinant human tissue-type plasminogen activator (rt-PA) in humans. J Pharmacol Exp Ther. 1985;235(2):506-12.

16. van Houwelingen RC, Luijckx GJ, Mazuri A, Bokkers RP, Eshghi OS, Uyttenboogaart M. Safety and Outcome of Intra-Arterial Treatment for Basilar Artery Occlusion. JAMA Neurol. 2016;73(10):1225-30.
17. Uno J, Kameda K, Otsuji R, et al. Mechanical Thrombectomy for Acute Basilar Artery Occlusion in Early Therapeutic Time Window. Cerebrovasc Dis. 2017;44(3-4):217-24.

18. Kang DH, Jung C, Yoon W, et al. Endovascular Thrombectomy for Acute Basilar Artery Occlusion: a Multicenter Retrospective Observational Study. J Am Heart Assoc. 2018;7(14).

19. Giorgianni A, Biraschi F, Piano M, et al. Endovascular Treatment of Acute Basilar Artery Occlusion: Registro Endovascolare Lombardo Occlusione Basilar Artery (RELOBA) Study Group Experience. J Stroke Cerebrovasc Dis. 2018;27(9):2367-74.

20. Ferbert A, Bruckmann H, Drummen R. Clinical features of proven basilar artery occlusion. Stroke 1990;21(8):1135-42.

21. Lindsberg PJ, Pekkola J, Strbian D, Sairanen T, Mattle HP, Schroth G. Time window for recanalization in basilar artery occlusion: speculative synthesis. Neurology 2015;85(20):1806-15.

22. Puetz V, Khomenko A, Hill MD, et al. Extent of hypoattenuation on CT angiography source images in basilar artery occlusion: prognostic value in the Basilar Artery International Cooperation Study. Stroke 2011;42(12):3454-9.

23. Puetz V, Sylaja PN, Hill MD, et al. CT angiography source images predict final infarct extent in patients with basilar artery occlusion. AJNR Am J Neuroradiol. 2009;30(10):1877-83.

24. Lee HM, Kim M, Suh SI, et al. Lesions on DWI and the Outcome in Hyperacute Posterior Circulation Stroke. Can J Neurol Sci. 2014;41(2):187-92.

25. Strbian D, Sairanen T, Silvennoinen H, Salonen O, Kaste M, Lindsberg PJ. Thrombolysis of basilar artery occlusion: impact of baseline ischemia and time. Ann Neurol. 2013;73(6): 688-94.

26. Nogueira RG, Jadhav AP, Haussen DC, et al. Thrombectomy 6 to 24 Hours after Stroke with a Mismatch between Deficit and Infarct. N Engl J Med. 2018;378(1):11-21. 